\title{
Experimental evidence for the presence of nonacoustic excitations in molten Ga
}

\author{
F. J. Bermejo and I. Bustinduy* \\ Dept.Electricity and Electronics, University of the Basque Country, P.O. Box. 644, Bilbao 48080, Spain \\ S. J. Levett and J. W. Taylor \\ Rutherford Appleton Laboratory, Chilton, Didcot Oxon OX11, OQX \\ R. Fernández-Perea and C. Cabrillo \\ Instituto de Estructura de la Materia, C.S.I.C., Serrano 123, Madrid 28006, Spain
}

(Received 26 May 2005; published 8 September 2005)

\begin{abstract}
The nature of the broad band of microscopic excitations measured in molten gallium is investigated by means of analysis of their mode eigenvectors $I(Q, \omega)$. The results, derived from neutron spectroscopy experiments, show that excitations with a dominant acoustic character are confined to low energy transfers. In contrast, those appearing at higher frequencies unmistakably exhibit optical character, as evidenced by the out-of-phase relationship shown by the phase of oscillations in $I(Q, \omega)$ that become significantly shifted relative to those of the static structure factor. Such effects are shown to arise from partial covalency that is already known to be present in gaseous, cluster and crystalline forms.
\end{abstract}

DOI: 10.1103/PhysRevB.72.104103

PACS number(s): 61.25.Mv, 61.10.Eq, 63.50.+x

\section{INTRODUCTION}

The microscopic dynamics of liquid alkali metals is widely accepted to constitute the closest physical realization of analytically workable models such as those cast in terms of hard-spheres (HS). ${ }^{1,2}$ Dynamically, such systems are understood at the qualitative level as harmonic liquids ${ }^{3}$ where the spectrum of collective excitations $S(Q, \omega)$ may be represented by Einstein-type oscillators with well defined frequencies $\omega_{Q}$. However, this picture is known to be far too primitive to describe a basic property of liquid semimetals $(\mathrm{Si}, \mathrm{Ge}$, $\mathrm{Ga}$ ), namely the static structure factor $S(Q)$. The main reason lays in the noticeable asymmetry shown by its first peak, which may transform into a well defined shoulder within the super-cooled liquid realm (see, for instance, Refs. 4 and 5). Such asymmetry points towards the presence of two relevant length-scales which, roughly stated, arise from the coexistence of metallic and partial-covalence effects ${ }^{6,7}$ and lead to the stabilization of clusters that melt at temperatures close to twice the value of the crystal melting. ${ }^{8}$ This interplay of forces of different origin translates into effective two-body potentials devoid of the long-ranged Friedel oscillations characteristic of molten alkali metals and in fact, such potentials derived from inversion of experimental $S(Q)$ 's are found to show shallow minima being positive (repulsive) within the domain of distances characteristic of the first-neighbors shell. The action of such potentials is best assessed by comparison of the complex phase diagram of semimetals to those for alkali-metals or rare gases. The latter retain cubic structures while the former exhibit, in the case of $\mathrm{Ga}$ a minimum of five phases below melting at ambient pressure $\left(T_{m}\right.$ $=302.93 \mathrm{~K}$ ), all of them displaying lower crystal symmetries. Such crystal phases exhibit, as expected, rather different vibrational spectra ${ }^{9}$ from those of cubic alkali-metals, displaying a number of low-lying optical branches.

Following the same line of reasoning employed for liquid alkali-metals, namely that leading to the expectancy of find- ing some remnants of the crystal phonons upon melting, here we pursue such an endeavor by means of measurements of the spectra of collective excitations $S(Q, \omega)$ for molten and crystalline $\alpha-\mathrm{Ga}$ through neutron spectroscopy. The measurement on $\alpha-\mathrm{Ga}$ is taken as the most significant reference state for the liquid.

\section{RESULTS}

\section{A. Experiment}

Here we report on two sets of measurements carried out on a Ga sample contained within a furnace or a closed cycle refrigerator using the MARI chopper spectrometer at the ISIS pulsed neutron source.

The instrument was setup allowing the exploration of $S(Q, \omega)$ surfaces using incident neutron energies $E_{i}=100 \mathrm{meV}[\Delta E \approx 1.2 \mathrm{meV}$ full width at half maximum (FWHM)] and $E_{i}=12 \mathrm{meV}(\Delta E \approx 0.43 \mathrm{meV}$ FWHM). The sample was contained within quartz tubes of $0.5 \mathrm{~cm}$ inner diameter. Measurements were made at temperatures close to melting at $T=315 \mathrm{~K}\left(T_{m}=302.93 \mathrm{~K}\right)$ as well as at $873 \mathrm{~K}$. The experimental double differential scattering cross sections were corrected for sample self-attenuation, multiplescattering and multi-excitation effects. ${ }^{10}$

An intensity plot of the $\omega(1-\exp (\beta \omega)) I(Q, \omega)$, with $\beta$ $=1 / k_{B} T$ surface from a measurement of the liquid close to melting is depicted in Fig. 1. Choosing this quantity rather than the bare $I(Q, \omega)$ was motivated by the absence of any truly remarkable feature in the latter. In turn, maxima of side peaks of $\omega(1-\exp (\beta \omega)) I(Q, \omega)$ can be taken as first hand indications of the presence of a broad band of excitations. There, the linear dispersion laws corresponding to extrapolations of hydrodynamic laws calculated using the macroscopic value of the isothermal sound velocity as well as those recently reported from neutron and inelastic x-ray stud- 

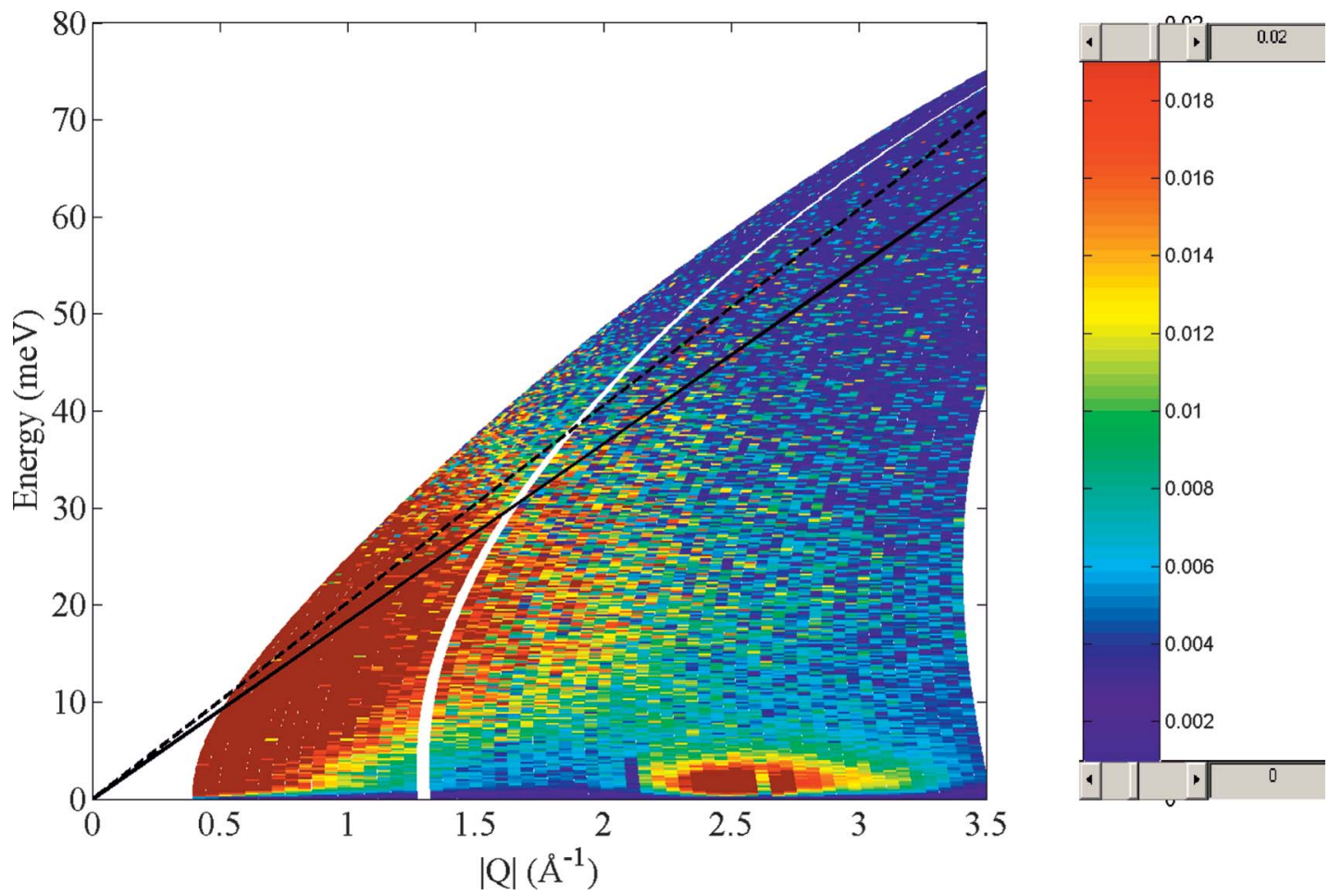

FIG. 1. (Color) Intensity plot for molten $\mathrm{Ga}$ at $T=315 \mathrm{~K}$ (see text). Straight solid line represent hydrodynamic dispersion following the isothermal value (Ref. 11). Dashed line depicts the dispersion using the sound velocity values from Ref. 12. Color code: From blue to red.

ies are also shown. ${ }^{12} \mathrm{~A}$ glance at Fig. 1 shows that a region of well defined excitations is found for energies below 30 meV and wave-vectors below some $1.3 \AA^{-1}$. Both extrapolated linear dispersions cross the region where intensities are maximal. There is however an additional detail that merits consideration. This refers to the presence of a prominant (light-blue) halo with somewhat less intensity but extending over a wider $Q-\omega$ region. In subsequent paragraphs we examine the significance of this feature.

Data measured using lower incident energies served to study in detail the quasielastic part of the spectrum. A representative sample of such spectra is shown in Fig. 2. There one can clearly see that this spectral region is characteristic of stochastic motions (i.e., those having an average zero frequency) and is composed of by at least two contributions. As we shall detail below, both components can be identified after modeling the spectrum as a sum of two lorentzians as an incoherent-scattering contribution that probes singleparticle liquid dynamics as well as a coherent-scattering counterpart that arises from correlated particle motions.

A computer molecular dynamics simulation was carried out employing the effective potential given in Ref. 7 resulting from a theoretical inversion of the experimental static liquid structure factor. While the results as we will show below clearly overemphasize the presence of collective excitations, the calculation provided us access to some quantities not easily attainable by experiment.

\section{B. Quasielastic spectra}

The intensity $I(Q, \omega)$ fulfills

$$
\mathrm{I}(Q, \omega) \propto S(Q, \omega) \otimes R(Q, \omega)
$$

$$
S(Q, \omega)=\frac{\sigma_{i}}{\sigma_{i}+\sigma_{c}} S_{i}(Q, \omega)+\frac{\sigma_{c}}{\sigma_{i}+\sigma_{c}} S_{c}(Q, \omega)
$$

where $R(Q, \omega)$ stands for the appropriate instrument resolution function that is convoluted with the total dynamic structure factor $S(Q, \omega)$ and $S_{i}(Q, \omega)$ and $S_{c}(Q, \omega)$ refer to its quasielastic and inelastic components weighted by their relative cross sections ( $\sigma_{i}=0.16$ barns, $\sigma_{c}=6.675$ barns).

A glance at the spectra shown in Fig. 2 unveils the presence of two quasielastic components having rather different linewidths for the lower two wave-vectors. The observed intensity can thus be well accounted for in terms of two Lorentzian functions for wave-vectors below $Q \approx 1.4 \AA^{-1}$ and with a single Lorentzian component beyond such wavevectors. The results concerning the quasielastic linewidths as well as their corresponding amplitudes are shown in Figs. 3 and 4.

Figure 3 displays results concerning the linewidths for both quasielastic components at the two measured temperatures. From the approximately Fickian dependence of such linewidths, that is $\Delta \omega(Q)=2 \hbar D_{s} Q^{2}$ estimates for the massdiffusion coefficient $D_{s}$ are obtained. The obtained values yield figures of $0.132 \AA^{2} \mathrm{meV}$ for $T=315 \mathrm{~K}$ and $0.737 \AA^{2} \mathrm{meV}$ for $T=873 \mathrm{~K}$, that are to be compared with reported values of $0.104 \AA^{2} \mathrm{meV}$ at melting ${ }^{13}$ and $0.862 \AA^{2} \mathrm{meV}$ at $T=970 \mathrm{~K}$ for the macroscopic selfdiffusion coefficient. Notice, however, that reports on measurements carried out by macroscopic means yield data with some $15 \%$ accuracy at their best.

The linewidths corresponding to the coherent scattering counterpart show strong wave-vector and temperature depen- 


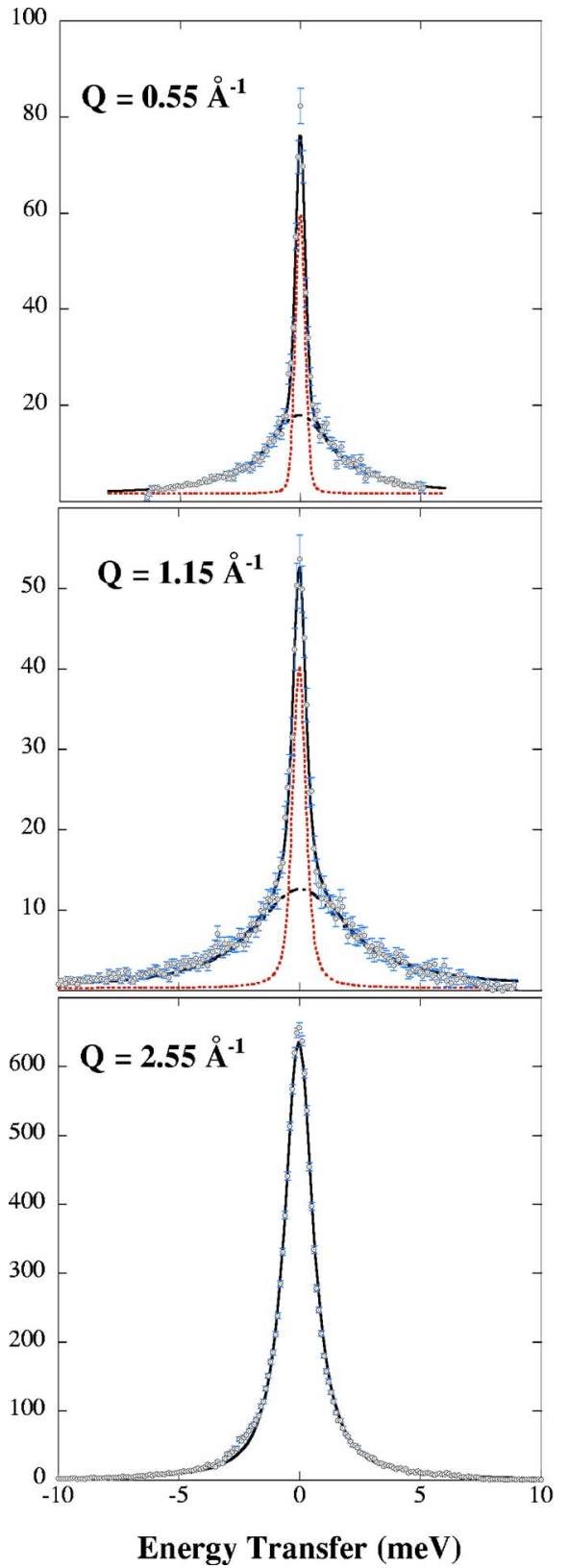

dences. The coherent spectral width may be described by means of the expression derived by Cohen et al. ${ }^{14}$ for a liquid composed by hard-spheres on the grounds of the Revised Enskog Theory (RET),

$$
\Delta \omega=D_{E} Q^{2} / S(Q)\left[1-j_{0}(Q \sigma)+2 j_{2}(Q \sigma)\right],
$$

with $D_{E}$ being the Enskog diffusion coefficient and $\sigma$ a corresponding hard-sphere diameter. The Eq. (2) is known to adequately reproduce data measured for blatantly Non-HardSphere systems ${ }^{15}$ such as liquefied rare gases, ${ }^{14}$ molten alumina or germanium, provided that some refined estimates for $D_{E}$ and $\sigma$ are chosen and used together with the experimental $S(Q)$. A test of the predictive capability of such an expression is provided by our estimate that is displayed in Fig. 3 for the lower temperature. These have been computed using two different $S(Q)$ s and both $D_{E}$ and $\sigma$ are taken from experiment as
FIG. 2. (Color online) Quasielastic scattering spectra for molten $\mathrm{Ga}$ at $T=315 \mathrm{~K}$ (left-side frames) and $T=873 \mathrm{~K}$ (right-side frames). Straight solid lines display fits to a model that comprises both incoherent and coherent intensities. Dotted and dashed lines depict the two Lorentzian components. Spectra shown for Q $=2.55 \AA^{-1}$ can be well accounted for using a single Lorentzian. 


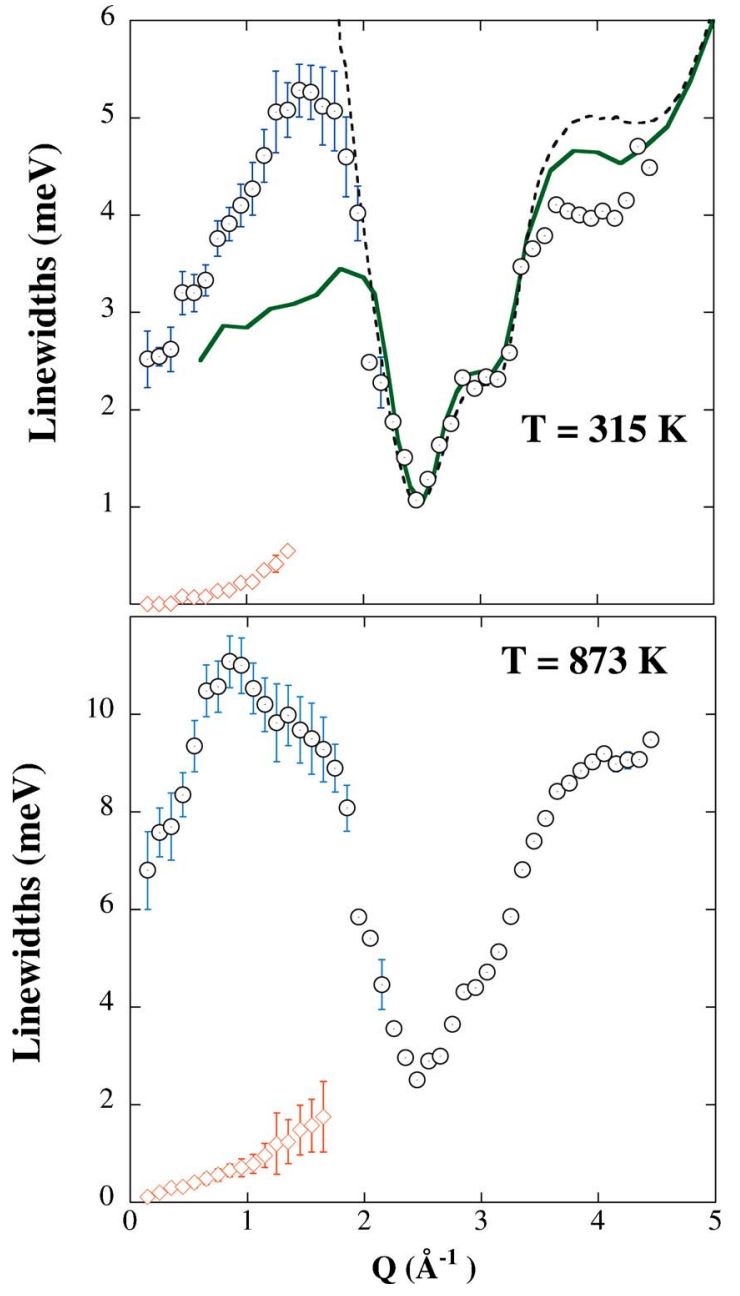

FIG. 3. (Color online) Quasielastic linewidths for molten Ga at $T=315 \mathrm{~K}$ (upper frame) and $T=873 \mathrm{~K}$ (lower frame). Open circles depict coherent linewidths and lozenges show the incoherent counterparts. Straight solid lines display predictions made using Eq. (2) using for the purpose experimental structure factors given in Refs. 16 and 7.

the coherent quasielastic widths of the liquid at $320 \mathrm{~K}$ and $970 \mathrm{~K}$ are reported to be independent of wavevector yielding widths of 6.7 and $11.2 \mathrm{meV}$, respectively. The physical mechanisms underlining such a spectral component are difficult to guess. However on the basis of Eq. (2) it seems clear that both mass-diffusion as well as thermal conduction effects give rise to such a spectral component.

The amplitudes of the two quasielastic components are shown in Fig. 4. Data concerning the narrower line shows, as expected a wave-vector dependence mainly governed by a $\propto 1 / D_{s} Q^{2}$ behavior plus mode-coupling terms. ${ }^{18}$ In contrast, the amplitudes for the coherent-scattering components closely follow the details of the static structure factors.

\section{Wave-vector dependence of inelastic intensities: Liquid}

For a molten material such as Ga which may show complex dynamics, the character of the observed excitations is best assessed on the basis of the Q-dependence of $S(Q, \omega)$

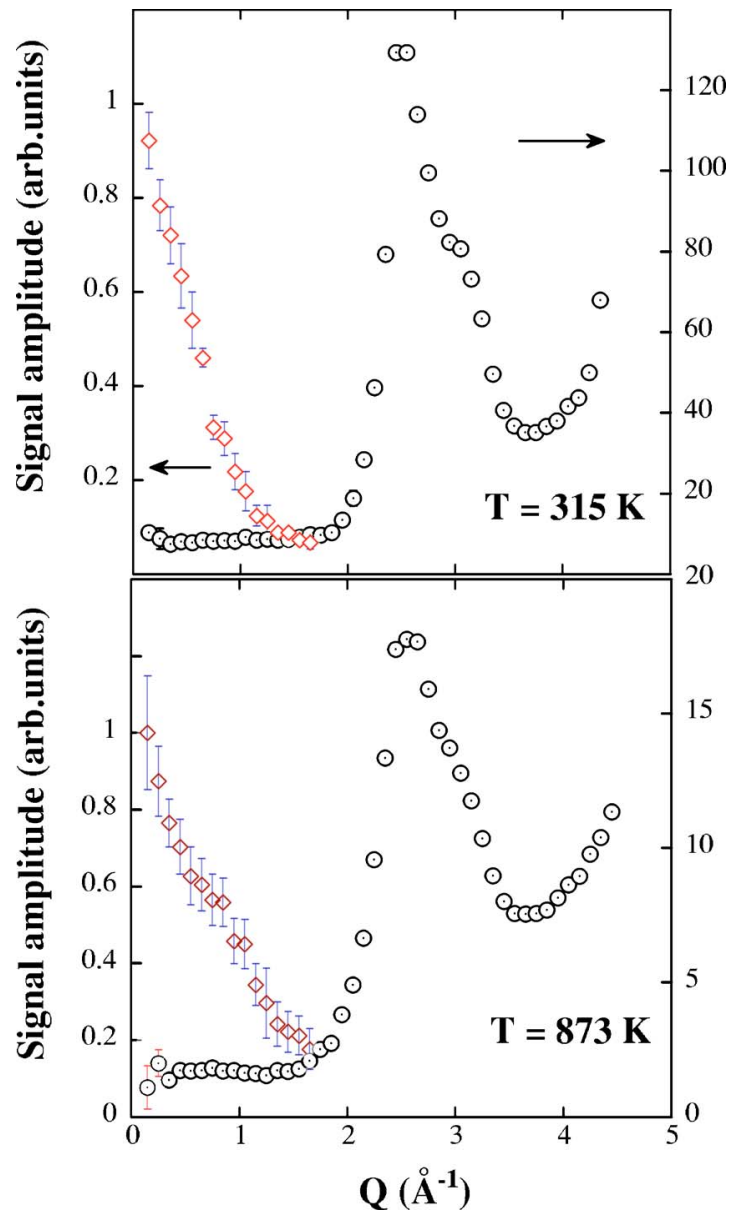

FIG. 4. (Color online) Amplitudes for the two quasielastic lines for molten $\mathrm{Ga}$ at $T=315 \mathrm{~K}$ (upper frame) and $T=873 \mathrm{~K}$ (lower frame). Open circles depict coherent linewidths and lozenges show the incoherent counterparts.

for a given energy transfer. These quantities provide an insight into the excitation form factors and thus serve to characterize the details of atomic motions.

Here, our point of departure will be to test, using our experimental data the behavior expected for a liquid which only sustains longitudinal collective excitations (i.e., density oscillations). Under such a consideration we expect the spectral intensities to follow wave-vector dependencies given by the reduced spectral moments,

$$
\omega_{0}^{2}=\frac{Q^{2}}{\beta M S(Q)}
$$

$\omega_{l}^{2}=\frac{3 Q^{2}}{\beta M}+\omega_{E}^{2}\left[1-\frac{3 \sin \left(Q R_{0}\right)}{Q R_{0}}-\frac{6 \cos \left(Q R_{0}\right)}{\left(Q R_{0}\right)^{2}}+\frac{6 \sin \left(Q R_{0}\right)}{\left(Q R_{0}\right)^{3}}\right]$

where $\beta=1 / k_{B} T, \omega_{E}=22.4 \mathrm{meV}$ plays the role of an Einstein frequency ${ }^{19}$ and $R_{0}=2.79 \AA$ represents the distance which approximately corresponds to the main minimum in the interaction potential. Both parameters have been determined from the inverted potential ${ }^{7}$ as well as from the molecular dynamics simulation referred to previously. The value for the 


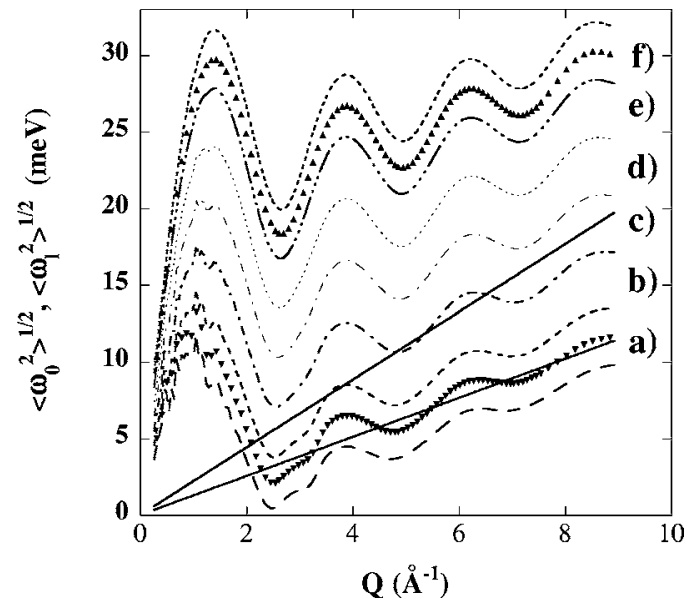

FIG. 5. A schematic representation of the low- and highfrequency limits used to calculate the inelastic form-factors for the liquid (see text). The inverted solid triangles depict the calculated values of $\sqrt{\omega_{0}^{2}}$, while the solid triangles show $\sqrt{\omega_{l}^{2}}$. The integration limits for each band (a)-(f) are shown by the pairs of dashed lines. That is, integrals calculated for the lowest energies [frame (a)] have lower and upper limits given by the dashed lines at both sides of $\sqrt{\omega_{0}^{2}}$, and those for the highest energies [frame (f)] are gives by dash-dot and short-dashed line at both sides of $\sqrt{\omega_{l}^{2}}$. Frames (b)-(e) comprise intermediate energies with limits for the integrals taken over energy transfers given by pairs of broken (dashed or dashdotted) lines. The solid lines depict the ideal-gas limits for the two reduced frequency moments, respectively.

former comes to be the same, as expected, as that where the experimental static pair distribution function shows its maximum.

For a mono-atomic simple liquid composed by particles interacting via spherically symmetric potentials the reduced spectral moments provide the low- and high-frequency limits of the excitation frequencies of collective particle motions within the liquid. For $Q \rightarrow 0$ they go to well known hydrodynamic limits given by the isothermal and high-frequency sound velocities respectively, while at large Q-values they approach the ideal-gas limits.

The paths to explore the $Q-\omega$ plane are thus set to those followed by the square roots of the reduced frequency moments $\sqrt{\omega_{0}^{2}}$ or $\sqrt{\omega_{l}^{2}}$. In doing so one explores the $Q-\omega$ plane along paths where the inelastic intensity is expected to be maximal. For this purpose we have divided the $Q-\omega$ into a number of sections as shown in Fig. 5. To account for the effects of finite lifetime of the excitations together with instrumental resolution and counting statistics, here we sample the inelastic intensities by integrating within finite intervals along bands of frequencies comprised within the aforementioned limits.

The results are depicted in Fig. 6. These correspond to integrals taken over the energy limits displayed in Fig. 5 taken at constant $Q$, to which a contribution to account for the $\mathrm{Q}^{2}$ dependence of the inelastic intensities (i.e., selfscattering not leading to interference effects) has been subtracted using for the purpose a previously reported model ${ }^{20}$

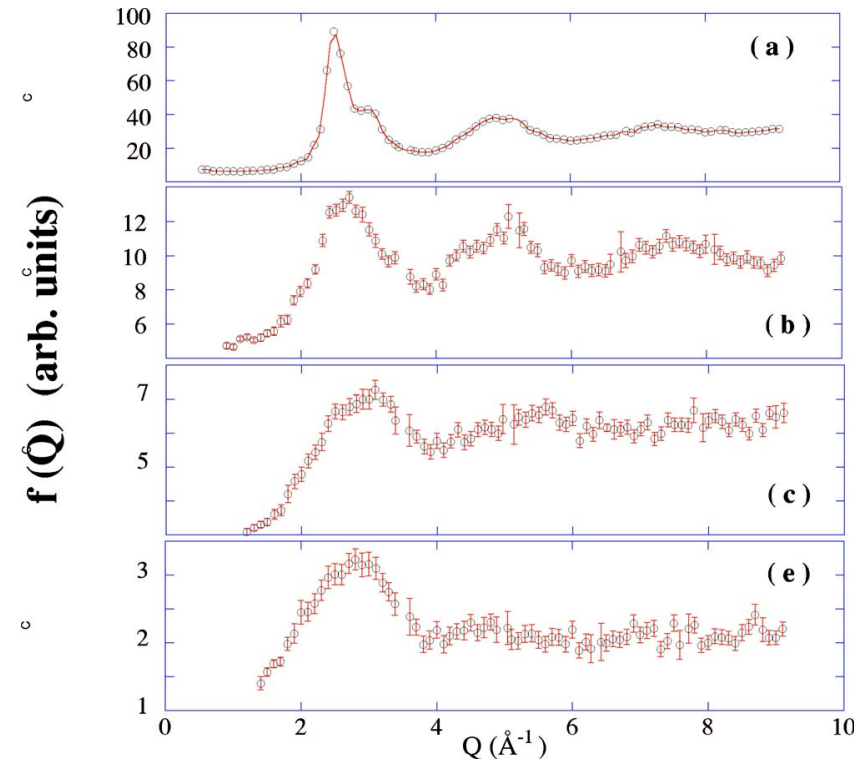

FIG. 6. (Color online) Inelastic intensity plots for liquid Ga corresponding to frequency regions shown in Fig. 1. Spectra have been corrected for the $Q^{2}$ pre-factors given in Eq. (5).

$$
S_{\text {self }}(Q)=A+B Q^{2} \exp \left(\frac{Q^{2} \gamma}{3}\right)
$$

where $A$ is a small background correction and the selfscattering term is defined by an amplitude $B$ and an approximation to the Debye-Waller factor given in terms of the rootmean-squared atomic displacements which are treated here as free parameters.

To gauge the meaning of graphs show in Fig. 6 let us recall some analytical results adequate for the description of the wave-vector dependence of a disordered body. For mostly elastic (or coherent quasielastic) scattering, that is at low energy transfers one expects the intensity not to deviate strongly from

$$
S_{c}(Q, \omega=0)=S_{e l}(Q) \delta(\omega) \approx \frac{1}{N} \sum_{j, j^{\prime}} j_{0}\left(Q d_{j, j^{\prime}}\right)
$$

where the summation runs over all pairs of atoms situated at distances $d_{j, j^{\prime}}, S_{e l}(Q) \propto S(Q)$ is the elastic structure factor and $j_{0}()$ is a spherical Bessel function. On the other hand, if the material can sustain $M$ excitations, each of them characterized by a spectrum $D_{i}(\omega)$ with average frequency $\bar{\omega}_{i}$, a structure factor can be written as ${ }^{21}$

$$
\begin{aligned}
S_{\text {inel }}(Q, \omega)= & \frac{1}{N} \sum_{j, j^{\prime}} \sum_{i=1, M} \frac{\hbar^{2} Q^{2} \operatorname{csch}\left(\hbar \omega / 2 k_{B} T\right)}{4 m \hbar \bar{\omega}_{i}} \\
& \times \sum_{i} D_{i}(\omega) F_{j, j^{\prime}}^{i}(Q),
\end{aligned}
$$

where $N$ is a normalization factor that stands for the number of particles, and $m$ refers to the mass of a $\mathrm{Ga}$ atom. The form-factors $F_{j, j^{\prime}}^{i}(Q)$ corresponding to the $i$ th excitation contain the relevant information on the geometry of atomic motions and may be calculated provided that detailed informa- 


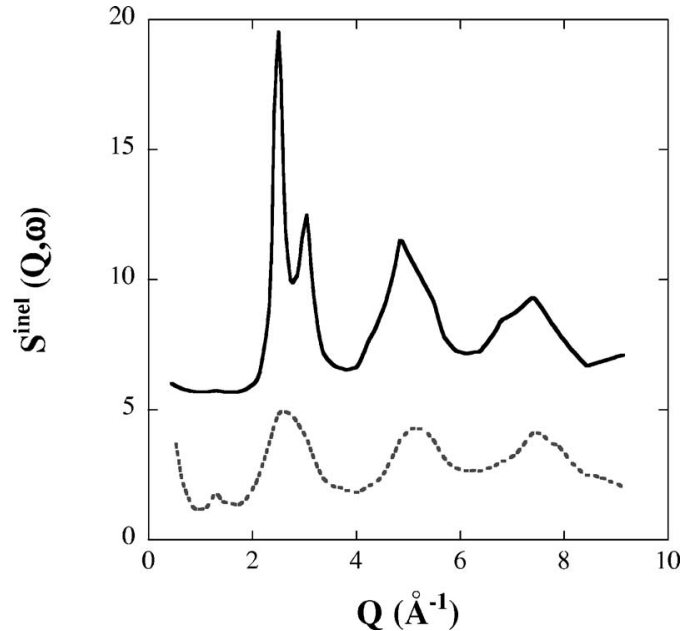

FIG. 7. Results from a calculation using Eq. (8) where the excitation frequencies are given by the square roots of both frequency moments. The upper trace corresponds to that for an excitation that follows the square root of the reduced zeroth frequency spectral moment $\sqrt{\omega_{0}^{2}}$, and that shown by dashes corresponds to that calculated for an excitation that follows $\sqrt{\omega_{l}^{2}}$. The latter curve has been magnified 20 times.

tion on the atomic vector displacements corresponding to every excitation is available. A simplified form for $S_{\text {inel }}(Q, \omega)$ can be written for the case of Brillouin scattering from longitudinal acoustic excitations traveling with velocity $v_{L}$ (i.e., assuming that the liquid can support collective density oscillations) and yields ${ }^{21}$

$$
\begin{aligned}
S_{\text {inel }}(Q, \omega)= & \frac{\hbar Q^{2}}{2 m} \frac{V}{8 \pi^{2} \omega} \operatorname{csch}\left(\frac{\hbar \omega}{2 k_{B} T}\right) \\
& \times\left[\frac{q_{L}^{2}}{v_{L}} f_{L}(Q, \omega)+4 \pi^{2} \rho \delta\left(\omega-v_{L} Q\right)\right]
\end{aligned}
$$

and

$$
f_{L}(Q, \omega) \simeq \int_{-1}^{1} \mu^{2} S\left(Q_{a v}, 0\right) d \mu, Q_{a v}=\left(Q^{2}+q_{L}^{2}-2 Q q_{L} \mu\right)^{1 / 2}
$$

Here $q_{L}=\omega / v_{L}$ and $V$ stands for the molar volume. The expression given by Eq. (8) can be calculated from the strictly elastic structure factor of the system as well as values for the longitudinal velocity as taken from from hydrodynamics. ${ }^{22}$ For the present case, a molecular dynamics simulation referred to above provided the required data. The results from such a calculation are shown in Fig. 7. There we see that the inelastic structure factor calculated along $\sqrt{\omega_{0}^{2}}$ closely resembles the calculated $S(Q)$, showing a well resolved secondary maximum at $\mathrm{Q} \approx 3 \AA^{-1}$. Data corresponding to an excitation following $\sqrt{\omega_{l}^{2}}$ still shows three well defined oscillations which still are in-phase with the main peaks of $S(Q)$.

A glance to results shown in Fig. 6 versus those shown in Fig. 7 reveals important differences especially for frequencies well above those given by $\sqrt{\omega_{0}^{2}}$. Both, experiment and simulation show at frequencies comparable to those of $\sqrt{\omega_{0}^{2}}$ inelastic intensities that show oscillations in-phase with the static liquid structure factor. Such a coincidence of phase shifts is, however, lost for higher frequencies. Indeed, the main peaks of Figs. 6(b)-6(e) shift from the position where $S(Q)$ shows its maximum $Q_{p}=2.48 \AA^{-1}$ to momentumtransfers of about $2.8 \AA^{-1}$ and continues shifting with increasing energy up to the band marked as Fig. 6(c) which now shows its main peak at $Q$ 's corresponding to those where the shoulder of the main peak of $S(Q)$ is located. Figure 6(e) corresponding to a band of frequencies about $\sqrt{\omega_{l}^{2}}$ now shows a single peak and, in stark contrast to that calculated from simulation it shows no further oscillations at higher momentum-transfers.

\section{Wave-vector dependence of inelastic intensities: Crystal}

To provide a reference to relate the findings for the melt, a measurement on polycrystalline $\alpha-\mathrm{Ga}$ was measured and inelastic structure factors were then calculated following procedures equivalent to those followed for the liquid. Energy slices of width $5 \mathrm{meV}$ have been used to carry out the integrations on horizontal bands, sampling different stripes along the phonon dispersion relations that are schematically shown in Fig. 8. The different paths to calculate the inelastic structure factor are chosen horizontally since contrary to the liquid where one expects the spectral moments to oscillate about the ideal-gas limits $\sqrt{\omega_{0 i d}^{2}}=Q^{2} / \beta M$ and $\lim _{Q \rightarrow \infty} \sqrt{\omega_{l i d}^{2}}$ $=3 Q^{2} / \beta M$ for momentum-transfers somewhat above $Q_{p}$, the data for a polycrystal will show nearly constant values past the first Brillouin zone.

The Fig. 9 displays a set of inelastic structure factors for the polycrystalline $\alpha-$ Ga for a set of energy-transfers matching that employed for the analysis of the melt data.

A comparison of Figs. 6 and 9 reveals the following. Much in the same way as observed for the liquid, the band corresponding to the lowest energy-transfers follows the elastic response. The lowest reflection from the crystal is observed at $Q=1.583 \AA^{-1}$ followed by an intense peak at $2.08 \AA^{-1}$. A far more drastic change is now observed for energy transfers within the range $5-10 \mathrm{meV}$ in the crystal than that seen for the liquid. In fact, the data in Fig. 9 shows that the complicated pattern seen at lower energies now transforms into a periodic oscillation with a first broad peak centered at about $3 \AA^{-1}$ and a period of $\approx 2.4 \AA^{-1}$. Such an oscillation persists up to energy transfers covered by the 10-15 meV band and diminishes at the higher energy band where only a broad peak centered at $3 \AA^{-1}$ remains.

\section{DISCUSSION}

\section{A. Model inelastic intensities}

The data displayed for polycrystalline $\alpha-\mathrm{Ga}$ shown in Fig. 9 can be compared with what may be expected for a vibration of nonacoustic origin within a macroscopically isotropic solid. On general grounds an inelastic structure factor for a vibration taken place at some frequency $\omega$ involving a pair of equal-mass atoms $i, j$ may be written in terms of the 


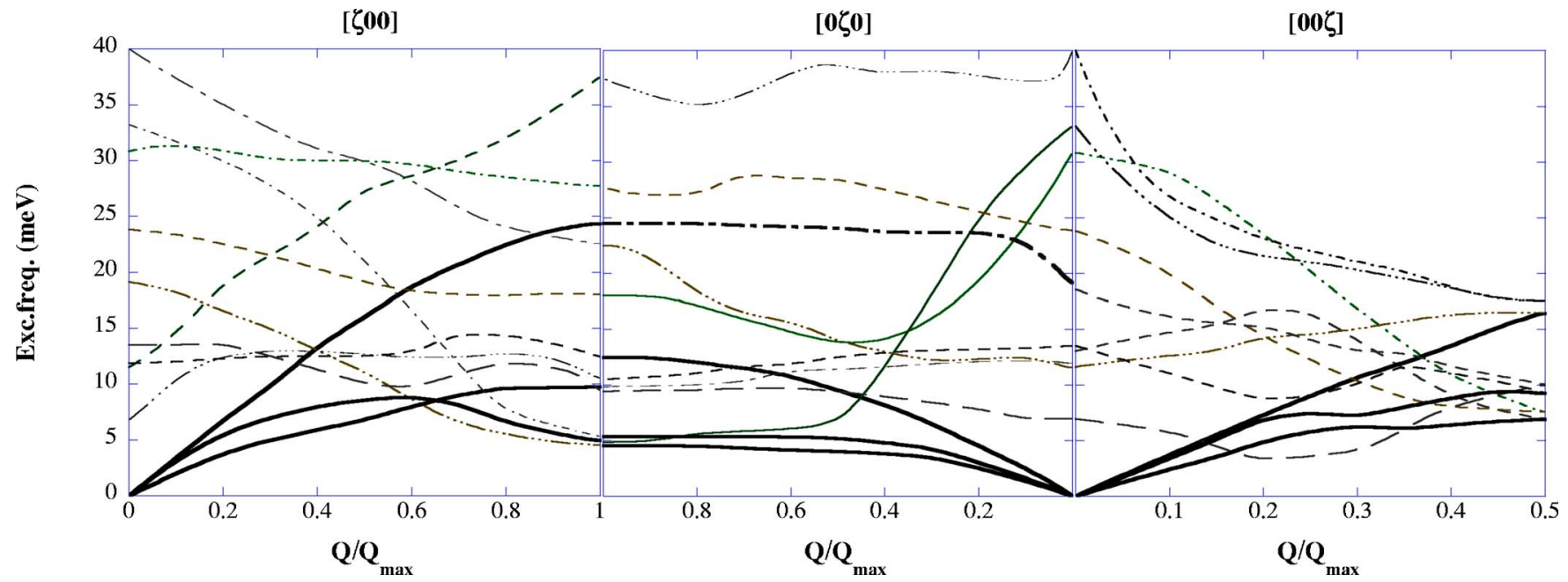

FIG. 8. (Color online) A schematic representation of the 12 phonon branches of crystalline $\alpha-$ Ga (Ref. 9), for the three directions of maximum symmetry.

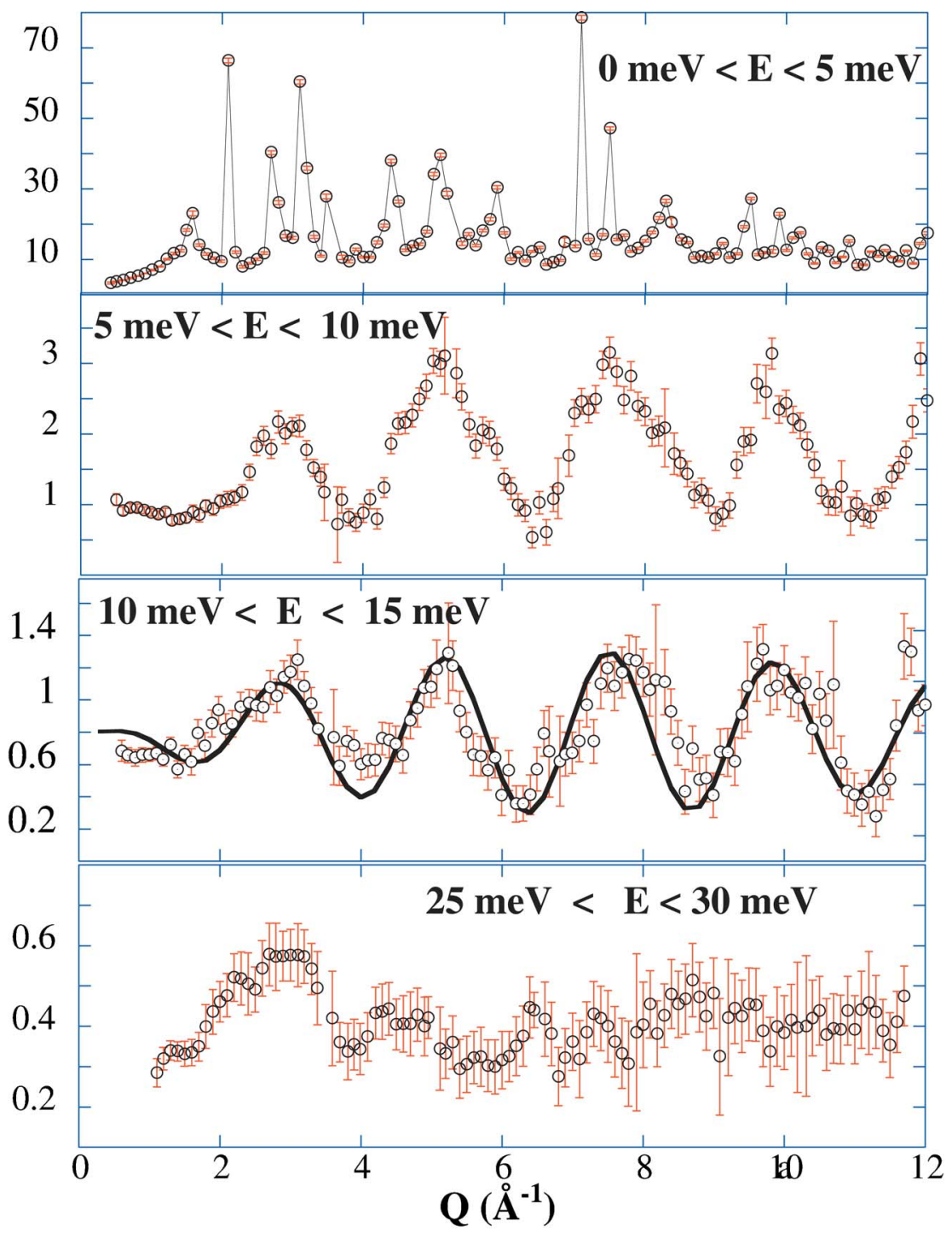

FIG. 9. (Color online) Inelastic intensity plots for polycrystalline Ga corresponding to frequency regions shown in Fig. 2. Spectra have been corrected for the $Q^{2}$ pre-factors given in Eq. (8). 
interatomic distances $\mathbf{R}_{\mathrm{ij}}$ and vector displacement vectors $\mathbf{e}_{\mathbf{i}}$ as $^{21}$

$$
\begin{aligned}
F^{\omega}(Q) / Q^{2}= & \frac{1}{m}\left[\frac{1}{3}\left(\mathbf{e}_{i} \cdot \mathbf{e}_{j}^{*}\right) j_{0}\left(Q R_{i j}\right)\right. \\
& \left.+\left(\frac{1}{3}\left(\mathbf{e}_{i} \cdot \mathbf{e}_{j}^{*}\right)-\frac{1}{R_{i j}^{2}}\left(\mathbf{R}_{i j} \cdot \mathbf{e}_{j}\right)\right) j_{2}\left(Q R_{i j}\right)\right]
\end{aligned}
$$

where $j_{0,2}()$ are spherical Bessel functions and bold-faced characters denote vector quantities. A direct calculation was carried out assuming purely out-of-phase vector displacements for a unit cell structure of $\alpha$-Ga which contains four atoms in its face-centered orthorhombic structure belonging to the $\mathrm{D}_{2 h}^{1} 8$ space group. Such an unusual metallic structure is shared by the solid halogens and indeed, the shortest atomic distance of $2.44 \AA$ is attributed to the presence of aggregates showing covalent character (dimers). At high temperatures ${ }^{23}$ its crystal structure shows that nearestneighbors of atoms joined by covalent bonds sit at distances of 2.71 and $2.80 \AA$, respectively. A calculation using Eq. (10) for the smaller distance and assuming that the displacement vectors correspond to purely anti-phase atomic motions and a root-mean-square displacement of $0.01 \AA^{2}$ as given by a recent study on premelting ${ }^{24}$ yields the solid curve depicted in Fig. 9. The comparison is suggestive of a strong contribution to the measured intensity from the nine modes of nonacoustic character that are pictorially shown in Fig. 8. In fact, from the crystal structure it is seen that atoms occupy Wyckoff sites with symmetry species $2 a_{g}+b_{1 g}+b_{2 g}+2 b_{3 g}$ $+a_{u}+b_{1 u}+b_{2 u}$ out of which $a_{g}+b_{1 g}+b_{2 g}+2 b_{3 g}$ are rotational in character and deemed to be of low frequencies. This is proven by the crystal dispersion relations shown in Fig. 8 where the nine optical branches are distributed within a frequency range of 5-40 meV and cross the three acoustic branches at relatively low points within the Brillouin zone. In consequence the comparison between experiment and calculation strongly suggests that most of the inelastic intensity above approximately $5 \mathrm{meV}$ for the polycrystalline solid arises from nonacoustic modes.

Melting of $\alpha-\mathrm{Ga}$ is sometimes assumed to lead to a purely metallic state devoid of covalence effects. ${ }^{12}$ This contrasts with recent data on gas-phase and small-cluster $\left(\mathrm{Ga}_{17}\right.$ and $\mathrm{Ga}_{13}$ ) samples ${ }^{8,25}$ that show the presence of strong covalency effects. It would therefore be surprising if such effects that are present within, gas, cluster and crystal phases were absent in the bulk liquid. Furthermore, reflectivity measurements on the liquid ${ }^{26}$ show surface-induced atomic layering that extends into the bulk liquid down to about three atomic diameters, which is indicative of strong directional interactions between $\mathrm{Ga}$ atoms.

At any rate, melting of $\alpha-\mathrm{Ga}$ is accompanied by an increase in electric resistivity not too dissimilar to others found for alkali-metals, ${ }^{27}$ the product of its thermal conductivity times resistivity at melting approaches the expected value for the Lorenz number and, remarkably enough, the volume coefficient of the electrical resistivity at melting, a property that

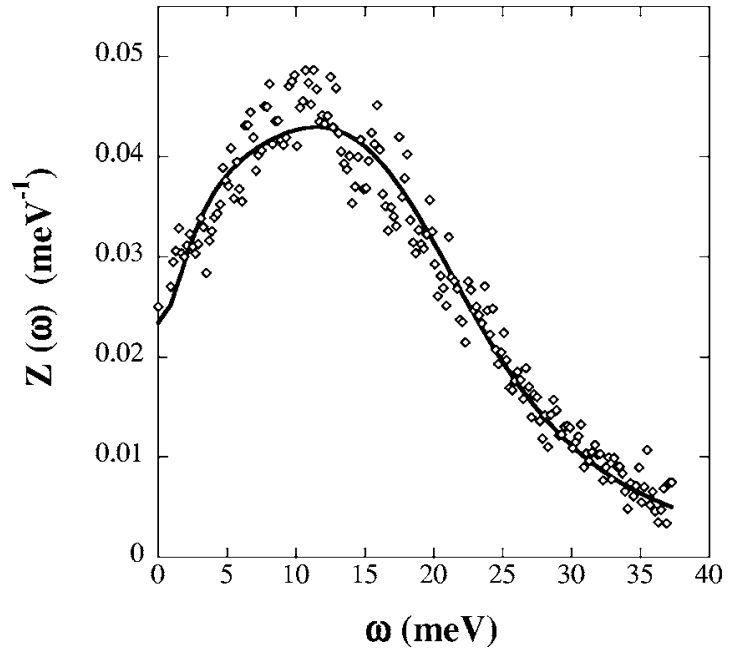

FIG. 10. An estimate for the spectral frequency distribution for liquid $\mathrm{Ga}$ at $T=315 \mathrm{~K}$. The distributions has been normalized to unit area.

explores changes in size of the Fermi sphere upon compression, locates molten Ga very close to semimetallic Bi and away from the alkali-metals.

The clearest proof of the competition between metallic and covalent interactions is given by the double peak structure shown in the main peak of $S(Q){ }^{4}$ This quantity has served to derive interatomic potentials ${ }^{5,7}$ which clarify the presence of electronic contributions significantly different from that corresponding to liquid metals, that may be assimilated to ensembles of hard-spheres. More specifically, such potentials show a change in curvature about the nearestneigbor distance, that precisely arise from a competition between hard-core and covalency-effects. In terms of microscopic atomic dynamics, the action of such potentials is known to enable the presence of Instantaneous Resonant Modes (IRM) ${ }^{28}$ these are quasilocalized modes that are created by a density oscillation which for the case in question (molten Ga at $T=1273 \mathrm{~K}^{28}$ ) have been predicted to occur with a density of modes showing a broad maximum at some 5-10 meV.

An estimate for the frequency spectrum calculated using procedures reported previously ${ }^{29}$ is shown if Fig. 10. In this case we use experimental data for $Q \leqslant 1.5 \AA^{-1}$ where coherent effects are deemed to be minimal.

The spectral distribution $Z(\omega)$ may be decomposed into an oscillatory part characterized by a single frequency $\omega_{o s c}$, a friction constant $\mu_{o s c}$, a parameter $a_{o s c}$ related to the oscillation lifetime and a stochastic part specified by the selfdiffusion coefficient and an additional friction term $\gamma_{r}$ that only affects the stochastic forces. The expression just referred to reads ${ }^{30}$

$$
\begin{aligned}
Z(\omega)= & \frac{2 M D}{\pi k_{B} T}\left[\frac{f^{2}}{\left(\omega^{2}+\mu_{o s c}^{2}\right) \omega_{o s c}^{2}+f^{2}}+\frac{f}{\omega_{o s c}^{2}} \frac{\omega^{2} a_{o s c}}{\omega^{2} \mu_{o s c}^{2}}\right] \\
& \times\left[\left(\frac{\omega^{2}-\omega_{o s c}^{2}}{\omega_{o s c}^{2}}\right)^{2}+\frac{\omega^{2} a_{o s c}^{2}}{\omega^{2}+\mu_{o s c}^{2}}\right]^{-1},
\end{aligned}
$$

with $f=\gamma_{r} \mu_{o s c}$, the the self-diffusion coefficients set to the 
macroscopic values and the rest of parameters left as adjustable. A parametric fit to the equation given above and the corresponding results shown in Fig. 10 yields best-fit values for the oscillation frequency $\omega_{o s c}=12.2 \mathrm{meV}$ that comes significantly below the estimates for the Einstein frequencies quoted above. Also, the estimate for $a_{\text {osc }}$ yields a value of 3.2 that comes between that of unity employed to fit the frequency distribution of liquid $\mathrm{Na}^{30}$ and that of 12 found adequate for liquid Ar. In other words, if a comparison is made with the clear-cut case of molten alkali-metals such as liquid $\mathrm{K}^{2}$, we find from the spectral distribution clear indications of relatively short lived oscillations as well as a large mismatch between the characteristic frequency $\omega_{\text {osc }}$ and that derived from the interaction potential that is identified as an Einstein frequency.

\section{B. Real space correlations}

A real space correlation function of the experimental inelastic structure-factors shown in Figs. 6 and 9 were calculated by means of sine Fourier transforms of the data. The results represent generalized real-space correlation functions $F(r)^{20}$ and are shown in Fig. 11.

Data shown in the upper frame of Fig. 11 compares the correlation function for the crystal to that of the liquid for the lowest energy transfers. Polycrystal data show a strong first peak centered about $2.71 \AA$ which, as already stated above, most probably arise from optic motions of a rotational kind. In contrast, the broad peak liquid for the liquid $F(r)$ is centered about $2.8 \AA$, a value that compares to that of $2.79 \AA$ where the static $g(r)$ shows its main maximum, which is the most probable distance between particles within the liquid. Such a coincidence attests the presence of a longitudinal acoustic excitation for such frequencies.

The lower frame shown in Fig. 11 displays how the maximum of $F(r)$ displaces towards smaller values as the energy transfer is increased. At the highest energy-transfer the maximum sits at distances of about $2.63 \AA$, a value that significantly exceeds that estimated for the Ga dimer bond-length $\left(2.444 \AA^{25}\right)$ but compares well with crystal data if the increase in density upon melting is taken into account. In other words, the closeness of the position of the main maximum of $F(r)$ shown by the crystal and liquid data at high energytransfers suggests that motions characterized in the crystal as optic-like should also be present within the liquid at high frequencies.

\section{CONCLUDING REMARKS}

The results reported here serve to illustrate the complex dynamics of molten $\mathrm{Ga}$ if compared to simpler metallic liquids. Our high-resolution measurement of the quasielastic spectrum, reveals the presence of a broad coherent quasielastic component showing a strong wave-vector and temperature dependences. Its linewidth comes about one order of magnitude larger than that found for molten potassium at an equivalent temperature. Understanding the origin of such different behaviors is still an unsolved issue within liquid state physics.

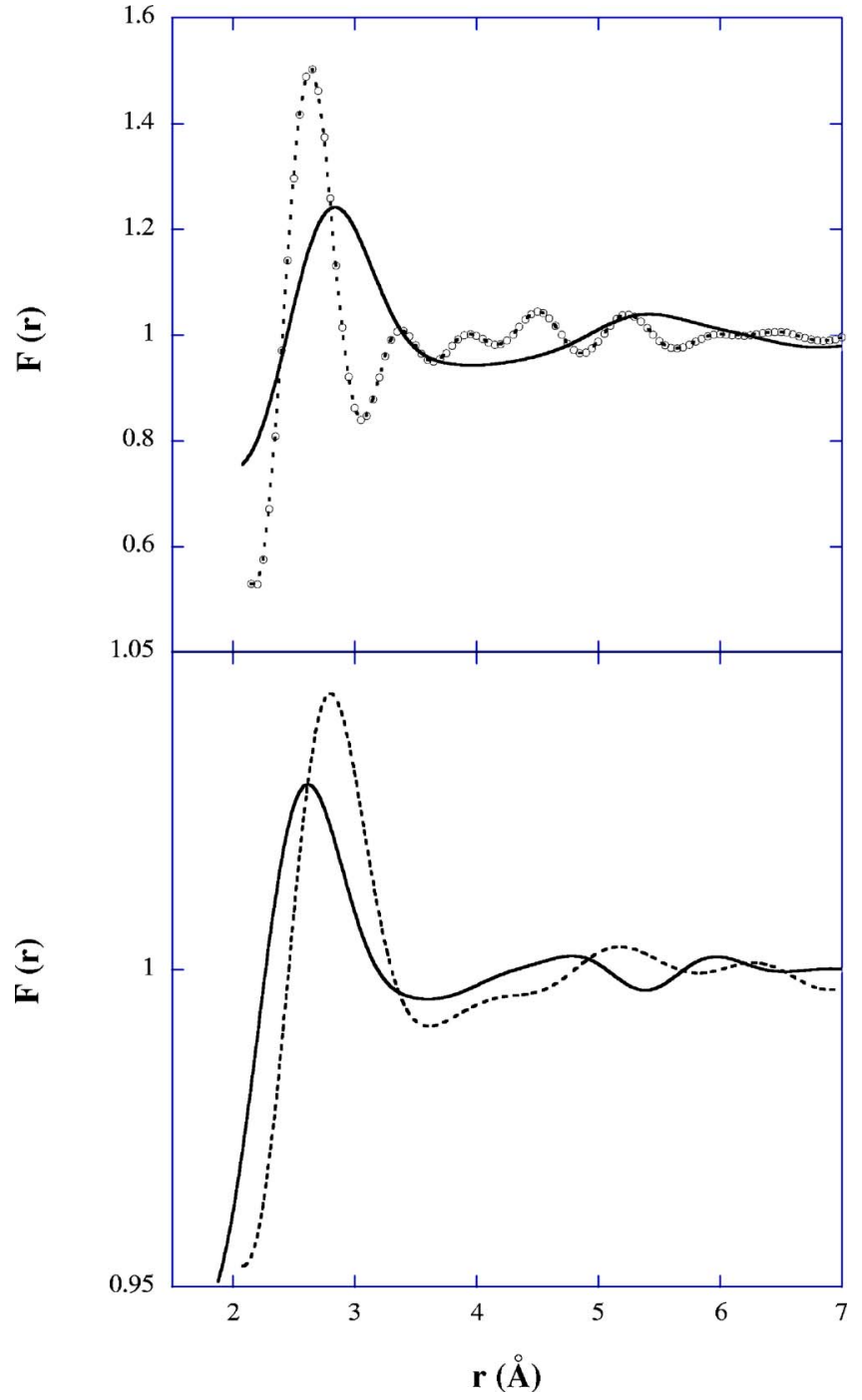

FIG. 11. (Color online) Real space correlation functions for a selection of the plots shown in Figs. 6 and 9. The upper frame shows a comparison of the transform for the crystal data (symbols) for 5-10 meV with that of the liquid for the lowest energy-transfers (solid-line). The lower frame shows the transforms corresponding to the bands (b) (dashes) and (e) for the liquid.

Our raw results are not dissimilar to others from a recent lower resolution neutron study reported by Bove et al. ${ }^{12}$ where clear inelastic side peaks are only observed after a large multiple scattering correction. Our main conclusions are however at odds with those derived by Bove et al. ${ }^{12}$ and also those reported in other studies performed using inelastic $\mathrm{x}$-ray scattering. ${ }^{12}$ In these previous studies data covering excitation energies up to $50 \mathrm{meV}$ are analyzed in terms of a single longitudinal excitation. In this latter respect, it is worth noting that the reported parameter values derived from analysis carried out under the latter assumption show discrepancies as large as $20 \%$ mostly due to difficulties in modeling the line shape of this complicated liquid. In addition, the interpretation of the derived frequencies in terms of a longitudinal acoustic mode is difficult to reconcile with the fact that such frequencies turn out to be larger within the 
melt than the orientational average of those observed for $\mathrm{Ga}$ in its $\alpha$ - or $\beta^{32}$ crystal forms.

In summary, our results give further support to the survival within molten $\mathrm{Ga}$ of strong directional effects usually attributed to competition between metallic and covalent bonding interactions. Our study thus comes into line with recent reports of similar findings in gaseous, ${ }^{25}$ cluster, ${ }^{8}$ liquid, , 6,33 and crystalline $9,31,32$ forms of this intriguing metal.

\section{ACKNOWLEDGMENT}

Work supported in part by Grant No. MAT2002-04540C05-03 (Spain).
*Electronic address: jbermejo@we.lc.ehu.es; Instituto de Estructura de la Materia, C.S.I.C., Serrano 123, Madrid 28006, Spain.

${ }^{1}$ N. H. March, Liquid Metals (Cambridge University Press, Cambridge, UK, 1990).

${ }^{2}$ See for instance C. Cabrillo, F. J. Bermejo, M. Alvarez, P. Verkerk, A. Maira-Vidal, S. M. Bennington, and D. Martin, Phys. Rev. Lett. 89, 075508 (2002);C. Cabrillo, F. J. Bermejo, A. MairaVidal, R. Fernandez-Perea, S. M. Bennington, and D. Martin, J. Phys.: Condens. Matter 16, S309 (2004).

${ }^{3}$ R. Eisenshitz and M. J. Wilford, Proc. Phys. Soc. London 80, 1078 (1962).

${ }^{4}$ S. F. Tsay and S. Wang, Phys. Rev. B 50, 108 (1994).

${ }^{5}$ S-F. Tsay and S. Wang, Phys. Rev. B 48, 5945 (1993).

${ }^{6}$ X. G. Gong, G. L. Chiarotti, M. Parinello, and E. Tosatti, Europhys. Lett. 21, 469 (1993).

${ }^{7}$ M. C. Bellissent-Funel, P. Chieux, D. Levesque, and J. J. Weis, Phys. Rev. A 39, 6310 (1989).

${ }^{8}$ G. A. Breaux, R. C. Benirschke, T. Sugai, B. S. Kinnear, and M. F. Jarrold, Phys. Rev. Lett. 91, 215508 (2003);S. Chacko, K. Joshi, and D. G. Kanhere, ibid. 92, 135506 (2004).

${ }^{9}$ W. B. Waeber, J. Phys. C 2, 903 (1969);W. Reichart, R. M. Nicklow, G. Colling, and H. G. Smith, Bull. Am. Phys. Soc. 14, 378 (1969);I. Spagnolatti and M. Bernasconi, Eur. Phys. J. B 36, 87 (2003).

${ }^{10}$ J. Dawidowski, F. J. Bermejo, and J. R. Granada, Phys. Rev. B 58, 706 (1998).

${ }^{11}$ In Ref. 13, p. 35.

${ }^{12}$ T. Scopigno, A. Filipponi, M. Krisch, G. Monaco, G. Ruocco, and F. Sette, Phys. Rev. Lett. 89, 255506 (2002);S. Hosokawa, W. C. Pilgrim, H. Sinn, and E. E. Alp, Physica B 350, 262 (2004);L. E. Bove, F. Formisano, F. Sacchetti, C. Petrillo, A. Ivanov, B. Dorner, and F. Barocchi, Phys. Rev. B 71, 014207 (2005).

${ }^{13}$ T. Iida and R. I. L. Guthrie, The Physical Properties of Liquid Metals (Oxford Science Publications, The Clarendon Press, Oxford (U.K.) 1993), p. 201.

${ }^{14}$ E. G. D. Cohen, P. Westerhuijs, and I. M. de Schepper, Phys. Rev. Lett. 59, 2872 (1987).

${ }^{15}$ See for instance H. Sinn, P. Glorieaux, L. Hennet, A. Alatas, M. Hu, E. E. Alp, F. J. Bermejo, D. L. Price, and M. L. Saboungi, Science 299, 2047 (2003);S. Hosokawa, Y. Kawakita, W. C. Pilgrim, and H. Sinn, Phys. Rev. B 63, 134205 (2001).

${ }^{16}$ D. I. Page, D. H. Saunderson, and C. G. Windsor, J. Phys. C 6,
212 (1973).

${ }^{17}$ T. Scopigno, R. Di Leonardo, L. Comez, A. Q. R. Baron, D. Fioretto, and G. Ruocco, Phys. Rev. Lett. 94, 155301 (2005).

${ }^{18}$ W. Montfrooy, I. de Schepper, J. Bosse, W. Glaser, and Ch. Morkel, Phys. Rev. A 33, 1405 (1986).

${ }^{19}$ S. W. Lovesey, Theory of Neutron Scattering from Condensed Matter (Oxford Science Publications, Oxford, 1984), Chap. 6.

${ }^{20}$ A. C. Hannon, M. Arai, and R. G. Delaplane, Nucl. Instrum. Meth. 354, 96 (1995).

${ }^{21}$ J. M. Carpenter and C. A. Pelizzari, Phys. Rev. B 12, 2391 (1975).

${ }^{22}$ M. Inui, S. Takeda, and T. Uechi, J. Phys. Soc. Jpn. 61, 3203 (1992).

${ }^{23}$ A. Defrain, J. Chim. Phys. Phys.-Chim. Biol. 74, 851 (1997).

${ }^{24}$ A. Rüm, H. Reichert, W. Donner, H. Dosch, Ch. Grütter, and J. Bilgram, Phys. Rev. B 68, 224110 (2003).

${ }^{25}$ G. S. Greetham and A. M. Ellis, J. Mol. Spectrosc. 222, 273 (2003);X. Tan and P. J. Dagdidian, J. Phys. Chem. A 107, 2642 (2003).

${ }^{26}$ M. J. Regan, E. H. Kawamoto, S. Lee, P. S. Pershan, M. Maskil, M. Deutsch, O. M. Magnussen, B. M. Ocko, and L. E. Berman, Phys. Rev. Lett. 75, 2498 (1995).

${ }^{27}$ F. J. Bermejo, L. Fernandez-Barquin, J. GarciaSoldevilla, D. Gomez-Plaza, and J. C. Gómez-Sal, Phys. Rev. E 50, 1341 (1994).

${ }^{28}$ T. M. Wu, S. F. Tsay, S. L. Chang, and W. J. Ma, Phys. Rev. B 64, 064204 (2001).

${ }^{29}$ M. Mukherjee, F. J. Bermejo, S. M. Bennington, and B. Fak, Phys. Rev. B 57, R11031 (1998).

${ }^{30}$ P. A. Egelstaff, An Introduction to the Liquid State (Oxford Science Publications, Oxford 1992), p. 252.

${ }^{31}$ M. I. Baskes, S. P. Chen and F. J. Cherne, Phys. Rev. B 66, 104107 (2002);M. Bernasconi, G. L. Chiarotti, and E. Tosatti, ibid. 52, 9988 (1995);X. G. Gong, G. L. Chiarotti, M. Parrinello, and E. Tosatti, ibid. 43, 14277 (1991).

${ }^{32}$ L. Bosio, R. Cortés, J. R. D. Copley, W. D. Teuchert, and J. Lefevre, J. Phys. F: Met. Phys. 11, 2261 (1981).

${ }^{33}$ F. J. Bermejo, M. Garcia-Hernandez, J. L. Martinez, and B. Hennion, Phys. Rev. E 49, 3133 (1994);F. J Bermejo, R. FernandezPerea, M. Alvarez, B. Roessli, H. E. Fischer, and J Bossy, ibid. 56, 3358 (1997). 